\title{
Lung Transplantation: A Systematic Review
}

\author{
Rodrigo Dominic Cerqueda Audirac ${ }^{1}$, Irving Kevi Moreno Valladares ${ }^{2}$, Alberto Robles Méndez \\ Hernández $^{3 *}$, Renata Buenfil Fuentes ${ }^{4}$, Oscar Alejandro Mora Torres ${ }^{5}$, Gabriel Isaac Castro \\ Enriquez $^{3}$, Erick Mauricio Aceves Rodríguez ${ }^{3}$, Bernardo Gutiérrez Muñoz ${ }^{3}$ \\ ${ }^{1}$ Facultad de Medicina, Universidad Nacional Autónoma de México \\ ${ }^{2}$ Department of General Surgery, Hospital San Angel Inn \\ ${ }^{3}$ Department of General Surgery, Hospital Angeles Metropolitano \\ ${ }^{4}$ Facultad de Medicina, Universidad Nacional Autónoma de México \\ ${ }^{5}$ Department of General Surgery, Hospital Angeles Lomas
}

\begin{abstract}
*Corresponding Author: Alberto Robles Méndez Hernández, Facultad Mexicana de Medicina, Universidad La Salle, Hospital Ángeles Metropolitano, Tlacotalpan \#59, Mexico City, Mexico, Email:albertormh01@gmail.com
\end{abstract}

\section{Abstract}

Introduction: Lung transplantation is the definitive treatment option for carefully selected patients with endstage lung disease, providing recipients with longer survival and improved quality of life.

Methods: The search terms were Lung Transplantation, a total of 11,445 articles were obtained, using the PubMed and Cochrane library search engines.

Results: The main indications for lung transplantation worldwide are:

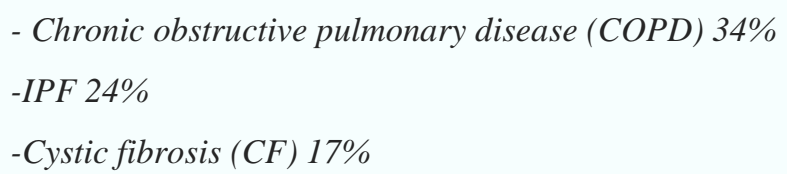

Only those patients who are ideally young, diagnosed with advanced lung disease and absence of disease in other organs and systems will be considered as candidates for lung transplantation.

Conclusions: Lung transplantation usually is the last therapeutic option for patients with respiratory failure. Despite many advances in immunology and the management of complications, the mortality and morbidity associated with this transplantation are far superior to others. Acute rejection is almost a universal problem in the first year, while bronchiolitis obliterans limits long-term survival. Respiratory infections also play an important role in the complications associated with lung transplantation due to the constant exposure of the graft to the external environment.

\section{Methodology}

The PubMed and Cochrane library search engines were used.

The filters used in PubMed were:

- Full text

- 5 years

The filters used in Cochrane were:

- All text

- 2 years

ARC Journal of Surgery
The search terms were Lung Transplantation, a total of 11,445 articles were obtained and after the inclusion and exclusion criteria, 19 were selected.

\section{- Definition}

Lung transplantation is the definitive treatment option for carefully selected patients with end-stage lung disease, which provides recipients with longer survival and improved quality of life. However, transplant survival is lower than desired due to its high mortality 
rate related to primary graft dysfunction, infection and chronic graft dysfunction, particularly in the form of bronchiolitis obliterans syndrome. [1]

\section{- Epidemiology}

According to the ISHLT (Internacional Society Heart and Lung Transplantation) the number of transplants has increased, reaching a total of 4127 in 2015.

Survival has also experienced an improvement at 1 and 5 years, being in $201380 \%$ and 54\%, reaching in 2015 $85 \%$ and $70 \%$ respectively.

Bronchiolitis Obliterans Syndrome (BOS) is responsible for $50 \%$ of late mortality. [2]

\section{- Indications}

The main indications for lung transplantation worldwide are:

- Chronic obstructive pulmonary disease (COPD) 34\%

-IPF $24 \%$

-Cystic fibrosis (CF) 17\%

-Alpha-1-antitrypsin deficiency (alpha1) $6 \%$

-Idiopathic Pulmonary Arterial Hypertension (IPAH) 3\%

-Lung fibrosis (not FPI) $4 \%$

-Bronchiectasis 3\%

-Retrasplantation $2.6 \%$

-Sarcoidosis 2, 5\%

Other indications for lung transplantation include connective tissue diseases, constrictive bronchiolitis, lymphangioleiomyomatosis, pulmonary hypertension secondary to congenital heart disease (in which the heart defect underwent a late correction or can be corrected at the time of transplantation), Langerhans cell histiocytosis, among others. [3]

- Candidate inclusion and exclusion criteria

\section{PATIENT SELECTION}

Candidates for lung transplantation are those patients with advanced and progressive lung disease, despite all clinical and surgical therapies, and who have a reduced life expectancy. In addition, candidates must demonstrate knowledge of the procedure, good compliance with the medical treatment provided and adequate psychosocial structure and family support.[3]

\section{- IDEAL DONOR}

Age $<55$ years old.

Smoking $<20$ packs/year.

Adequate weight/height matching with the receiver.

$\mathrm{PaFi}>300 \mathrm{mmHg}$ (With $100 \% \mathrm{FiO} 2$ and PEEP 5mmHg).

No evidence of active infection (clean bronchoscopy)

ABO compatibility (blood group).

Absence of thoracic trauma.

- No history of cardiac or pulmonary surgeries. [2]

\section{ABSOLUTE CONTRAINDICATIONS}

- Recent malignancy (other than nonmelanoma skin cancer)

- Active infection with hepatitis B or $\mathrm{C}$ virus associated with histologic evidence of significant liver damage

- Active or recent cigarette smoking, drug abuse, or alcohol abuse

- Severe psychiatric illness

- Repeated noncompliance with medical care

- Absence of a consistent and reliable social support network

- Infection with HIV is still viewed by most centers as an absolute contraindication, but promising results with liver, kidney, and heart transplantation in HIV-positive recipients, and a recent case report of successful lung transplantation, may soon remove this barrier

- The presence of significant extrapulmonary vital organ dysfunction precludes isolated lung transplantation, but multiorgan procedures such as heart-lung or 
lung-liver can be considered in highly select patients

- Both obesity and under- weight nutritional status increase the risk of post-transplant mortality, but cutoffs for exclusion of candidates vary among centers.[4]

\section{RELATIVE CONTRAINDICATIONS}

- Age > 65 years old.

- Chronic hepatitis B, C and HIV infection (in some international centers).

- IMV or ECMO.

- Poor rehabilitation potential.

- Colonization by highly resistant germs: bacteria, fungi and mycobacteria.

- $\quad>30$ and < 17 BMI.

- Severe symptomatic osteoporosis.

- Other untreated medical conditions: peptic ulcer, GERD, coronary artery disease. [2]

\section{DONOR}

Donors after brain death represent the traditional and largest source of lung donors. Another donor source that is increasingly being used to help meet the persistent shortage of lung donation is donation after circulatory death, a source that remains considerably underutilized but could significantly increase the donor lung pool, especially when combined with donor lung resuscitation and evaluation by ex vivo lung perfusion. [5]

\section{- PROCESS}

\section{IMMUNOSUPRESSION}

- Induction Therapy: It is applied at the time of transplantation and IL2 receptor antagonists (Basiliximab) predominate. In cases of highly sensitized patients: thymoglobulin, plasmapheresis, gamma globulin, rituximab, alemtuzumab, among others, can be used.

- Maintenance therapy: It is based on triple therapy: corticosteroids, calcineurin inhibitors (CNI) that block $\mathrm{T}$ lymphocyte activation: cyclosporine and tacrolimus (currently preferred) and antimetabolites that inhibit the proliferation of $\mathrm{B}$ and $\mathrm{T}$ lymphocytes: azathioprine and mycophenolate mofetil. [6]

\section{PROPHYLAXYS}

- Pneumocystis jirovecci and Nocardia sp: Cotrimoxazole Forte 1 tab three times a week and daily use for risk patients.

- Aspergillus sp: One-month nebulized amphotericin B associated with itraconazole or voriconazole in highrisk patients for 6-12 months. Requires strict monitoring of ICN levels. Herpes: Acyclovir for 4-6 months.

- Tuberculosis: In patients with PPD $>8$ $\mathrm{mm}$, or quantiferon (+) or at risk, HINRifampicin for 9 months pretransplant.

- CMV: If the recipient is $\operatorname{IgG}(-)$, valganciclovir is indicated for six months with monthly CMV PCR (polymerase chain reaction) controls. If the recipient is $\operatorname{IgG}(+)$, preventive therapy is performed and CMV PCR is measured biweekly for four months, and in case of increased immunosuppression, coverage with valganciclovir is performed. [7]

\section{- Technique}

- Monopulmonary or unipulmonary: it is the preferred choice in patients aged > 55 years and carriers of non-septic pathology. It facilitates the use of two single donor lung grafts. [8]

- The replacement of one or the other lung is technically indifferent, always replacing the one with the lowest perfusion.

- The patient is given arterial and venous lines, as well as a Swan-Ganz catheter, to monitor pulmonary artery pressures. Intubation will be selective in all cases.

- A standard posterolateral thoracotomy is performed through 5th space. The pulmonary hilum is exposed and the pulmonary artery is clamped for 5 minutes, if there are no hemodynamic disturbances or these are reversed with the administration of vasoactive drugs, dissection is continued, otherwise a 
partial femoro-femoral by-pass is established or cannulating aorta and right atrium, if the thoracotomy is on this side. Extrapericardial pneumonectomy is then performed, leaving long vascular pedicles, and the bronchus is sectioned with minimal prior dissection, to preserve bronchial circulation at this level.

- In telescoped anastomosis, loose mattress stitches placed in the cartilaginous zone allow intussusception of the smaller bronchus, usually the donor bronchus, into the recipient bronchus.

- Opening of the pericardium allows placement of a clamp into the recipient atrium, then resecting the proximal venous stumps and the existing bridge between the two veins, thus creating a wide left cuff.

- The anastomosis between the donor and recipient atrium was performed using a continuous 4/0 Prolene suture.

- The anastomosis of the pulmonary artery is performed end-to-end, with the same suture material as that used in the atrium, making sure that both the donor and recipient arteries are of adequate length; excessive length of one or the other could cause twisting of the anastomosis.

- Prior to organ reperfusion, $500 \mathrm{mg}$ of methylprednisolone is administered intravenously. Next, both lungs are ventilated and the clamp placed in the artery is gradually opened, causing hemorrhage at the level of the open anastomosis in the atrium, to purge the airway. Once this is completed, the atrial anastomosis is tied off.

- The thoracotomy is closed with the usual technique over two drains. At the end of the operation, bronchofibroscopy is performed to aspirate secretions and review the bronchial anastomosis.[9]

- Bipulmonary: It is the technique in increase worldwide, indicated in septic pathology, < 55 years old patients, advanced COPD and severe pulmonary hypertension. [10]
- With the patient in the supine position, a bilateral anterior thoracotomy is performed through the 4th space, with transverse section of the sternum. Dissection of the lower perfused lung is initiated first, in order to avoid by-pass, whenever possible. If clamping the pulmonary artery on this side causes hemodynamic instability, we will establish a partial by-pass by cannulating the right atrium and aorta, a maneuver that is usually necessary in $30 \%$ of cases. Pneumonectomy and graft implantation is performed according to the procedure described for single-lung transplantation.

- Once the anastomoses are completed, both lungs are ventilated, followed by selective ventilation of the newly implanted lung, contralateral pneumonectomy and implantation of the second graft, according to the technique already described. The thoracotomy is closed in the usual way and the sternum with steel stitches.[9]

- Cardiopulmonary: was the initial technique for lung transplantation, but is currently in disuse. It is only applicable in some centers, when there is congenital cardiovascular pathology associated with severe pulmonary hypertension.

\section{- Complications: Early and late}

\section{EARLY}

\section{- Primary graft dysfunction:}

Primary graft dysfunction (PGD) describes a form of acute allograft injury characterized by development of noncardiogenic pulmonary edema within 72 hours of transplantation in the absence of identifiable secondary causes. PGD is presumed to be a consequence of ischemia-reperfusion injury, but inflam- matory events associated with donor brain death, surgical trauma, and lymphatic disruption may be contributing factors. Treatment of severe PGD is supportive, relying principally on lowstretch mechanical ventilatory strategies. Under some 
circumstances, independent lung ventilation, extracorporeal life support, or inhaled nitric oxide can successfully stabilize critically ill patients. [11]

\section{- Infections:}

- Infection is an ever-present threat to the lung transplant recipient and a leading cause of both early and late deaths. A comprehensive discussion of infectious complications is beyond the scope of this article; only the most common pathogens are discussed. Bacterial pneumonia is by far the most frequently encountered infection, with a peak incidence in the first posttransplant month

- Fungal infections, especially Aspergillus sp, are very common, the frequent site of infection being the bronchial anastomosis.

- Some form of fungal prophylaxis is recommended for at least the first three months after transplantation.

- Cytomegalovirus (CMV) is the most common viral pathogen encountered after lung transplantation. Even with standard prophylactic measures, up to one-third of at-risk lung transplant recipients develop CMV disease within the first year [12]

- There is consensus in the international literature to prescribe CMV prophylaxis in the first three months of lung transplantation (except for IgGnegative donors with IgGnegative recipients); the benefit of extending it for six months and up to one year is debatable in cases of more severe viral disease risk (IgG-positive donor with IgG-negative recipient). [13]

- Acute cellular rejection: This refers to the immune response of the recipient, predominantly $\mathrm{T}$ lymphocytes, to the various antigens of the donor, which causes an inflammatory reaction of the pulmonary graft to a variable degree. It is frequent during the first post-transplant year (incidence up to 65\%) and does not normally represent an immediate risk for the patient. The diagnosis is made by transbronchial biopsy. Treatment consists of high doses of corticosteroids for 3 to 5 days and, in refractory cases, thymoglobulin. Acute cellular rejection is prevented with a combination of three drugs: corticosteroids, a calcineurin inhibitor (cyclosporine or tacrolimus) and a cell cycle inhibitor (azathioprine or mycophenolate) and should be maintained for the rest of the patient's life, unless there is some type of complication that justifies its suspension. [14]

\section{LATE COMPLICATIONS}

- Chronic graft dysfunction (CGD): It is characterized by progressive and irreversible deterioration of pulmonary function. It is the complication that determines the highest late morbidity and mortality. The causes are variable: from the presence of IPD in the immediate post-transplant period, then from the immunological point of view the development of repeated acute rejection and the formation of specific donor antibodies. Non-immunologic factors related are CMV infection, colonization and infection with Pseudomonas sp and of great importance in recent years the presence of gastroesophageal reflux. [15]

- Medical comorbidity: The long-term use of immunosuppressants facilitates cardiovascular pathology (hypertension and coronary 
artery disease), renal and metabolic dysfunction in a group of patients who must be maintained on treatment to avoid damage to other organs. [16]

- Neoplasms: Appearance of cutaneous malignant lesions, increased risk of lymphoproliferative syndrome and solid organ neoplasms, so endoscopic and imaging surveillance should be maintained. [17]

\section{- Prognosis}

As recorded in the ISHLT Registry, the overall median survival for the 24,936 patients who received a lung transplant between 1994 and 2008 was 5.3 years (2). Median survival has improved over time, from 4.0 years in the 1988 to 1994 era to 5.7 years in the 2000 to 2008 era. In the latter era, survival rates were $79 \%$ at 1 year, $63 \%$ at 3 years, $52 \%$ at 5 years, and $29 \%$ at 10 years. Disease-specific differences in survival are apparent but may be confounded by differences in severity of illness, comorbidities, and average age among these populations. In descending order, median survival is 7.1 years for $\mathrm{CF}$, 6.1 years for a1-antitrypsin deficiency, 5.2 years for COPD, 5.1 years for sarcoidosis, 4.6 years for IPAH, and 4.3 years for IPF. [18]

\section{CONCLUSIONS}

Lung transplantation usually is the last therapeutic option for patients with respiratory failure. Despite many advances in immunology and the management of complications, the mortality and morbidity associated with this transplantation are far superior to others. Acute rejection is almost a universal problem in the first year, while bronchiolitis obliterans limits long-term survival. Respiratory infections also play an important role in the complications associated with lung transplantation due to the constant exposure of the graft to the external environment.

\section{REFERENCES}

[1] Giménez-Milà M, Videla $S$, Pallarés N, Sabaté A, et al. Impact of surgical technique and analgesia on clinical outcomes after lung transplantation: A STROBE-compliant cohort study. Medicine (Baltimore). 2020 Nov 13;99(46):e22427.

[2] C. MTP, L. CS. TRASPLANTE PULMONAR: ESTADO ACTUAL. Rev Médica Clínica Las Condes. 2015 May;26(3):367-75.

[3] Afonso Júnior JE, Werebe E de C, Carraro RM, et al. Lung transplantation. Einstein São Paulo. 2015 Jun;13(2):297-304.

[4] Stehlik J, Edwards LB, Kucheryavaya AY, et al. The Registry of the International Society for Heart and Lung Transplantation: twentyseventh official adult heart transplant report2010. J Heart Lung Transplant. 2010;29(10):1089-103.

[5] Meyer KC. Recent advances in lung transplantation. F1000Research. 2018;7.

[6] Scheffert JL, Raza K. Immunosuppression in lung transplantation. J Thorac Dis. 2014;6(8):1039.

[7] Husain S, Zaldonis D, Kusne S, et al. Variation in antifungal prophylaxis strategies in lung transplantation. Transpl Infect Dis. 2006;8(4):213-8.

[8] Roman A, Morell F, Astudillo J, Margarit C, Bravo $\mathrm{C}$, et al. Unilateral lung transplantation: the first 2 cases. Group of Lung Transplantation of the University General Hospital of the Vall d'Hebron. Med Clin (Barc). 1993;100(10):3803.

[9] Hayanga JA, D'Cunha J. The surgical technique of bilateral sequential lung transplantation. J Thorac Dis. 2014;6(8):1063.

[10] Minces LR, Bhama JK, Abdel-Massih R, et al. Successful double lung transplantation in a patient with bilateral pulmonary and sinus aspergillomas. Transpl Infect Dis. 2011;13(5):485-8.

[11] Hadjiliadis D, Steele MP, Chaparro C, et al. Survival of lung transplant patients with cystic fibrosis harboring panresistant bacteria other than Burkholderia cepacia, compared with patients harboring sensitive bacteria. J Heart Lung Transplant. 2007;26(8):834-8.

[12] Kotloff RM, Thabut G. Lung transplantation. Am J Respir Crit Care Med. 2011;184(2):159_ 71.

[13] Aguilar-Guisado M, Givalda J, Ussetti P, et al. Pneumonia after lung transplantation in the RESITRA Cohort: a multicenter prospective study. Am J Transplant. 2007;7(8):1989-96. 
[14] Frost AE, Jammal CT, Cagle PT. Hyperacute rejection following lung transplantation. Chest. 1996;110(2):559-62.

[15] Whitson BA, Prekker ME, Herrington CS, et al. Primary graft dysfunction and long-term pulmonary function after lung transplantation. $\mathrm{J}$ Heart Lung Transplant. 2007;26(10):1004-11.

[16] Kreider M, Kotloff RM. Selection of candidates for lung transplantation. Proc Am Thorac Soc. 2009;6(1):20-7.
[17] Delgado M, Fernandez R, Paradela M, et al. Development of neoplasms during lung transplantation follow-up. In: Transplantation proceedings. Elsevier; 2008. p. 3094-6.

[18] Le Pavec J, Hascoët S, Fadel E. Heart-lung transplantation: current indications, prognosis and specific considerations. $J$ Thorac Dis. 2018;10(10):5946.

Citation: Rodrigo Dominic Cerqueda Audirac, Irving Kevi Moreno Valladares, Alberto Robles Méndez Hernández, Renata Buenfil Fuentes, Oscar Alejandro Mora Torres, Gabriel Isaac Castro Enriquez, Erick Mauricio Aceves Rodríguez, Bernardo Gutiérrez Muñoz. "Lung Transplantation: A Systematic Review”. ARC Journal of Surgery. 2021; 7(2):6-12. DOI: https://doi.org/ 10.20431/2455-572X.0702002.

Copyright: (C) 2021 Authors. This is an open-access article distributed under the terms of the Creative Commons Attribution License, which permits unrestricted use, distribution, and reproduction in any medium, provided the original author and source are credited. 